\title{
Increased Risk of Pneumonia among Patients with Inflammatory Bowel Disease
}

\author{
Millie D. Long, MD, MPH ${ }^{1,2}$, Christopher Martin, MSPH ${ }^{1,2}$, Robert S. Sandler, MD, MPH ${ }^{1,2}$, \\ and Michael D. Kappelman, MD, $\mathbf{M P H}^{2,3}$ \\ ${ }^{1}$ University of North Carolina at Chapel Hill, Department of Medicine, Division of \\ Gastroenterology and Hepatology \\ ${ }^{2}$ Center for Gastrointestinal Biology and Disease, Chapel Hill, NC \\ ${ }^{3}$ University of North Carolina at Chapel Hill, Department of Pediatrics, Division of \\ Gastroenterology and Hepatology
}

\section{Abstract}

Background \& Aims-Patients with inflammatory bowel disease (IBD) may be at increased risk for infectious complications. We aimed to determine the risk of pneumonia in IBD and how biologic and immunosuppressive medications affect this risk.

\begin{abstract}
Methods-We performed a retrospective cohort and a nested case-control study using administrative data from IMS Health Inc, LifeLink ${ }^{\mathrm{TM}}$ Health Plan Claims Database. In the cohort, IBD patients were matched to 4 individuals without IBD. Pneumonia risk was evaluated by incidence rate ratio (IRR) and by adjusted Cox proportional hazards models. In the nested casecontrol analysis, 4856 IBD patients with pneumonia were matched to up to 4 IBD patients without pneumonia by incidence density sampling. Conditional logistic regression was used to determine associations between medications and pneumonia.
\end{abstract}

Results-The cohort included 50,932 patients with Crohn's disease (CD), 56,403 patients with ulcerative colitis (UC), and 1,269 with unspecified IBD; matched to 434,416 individuals without IBD. The IBD cohort had an increased pneumonia risk when compared to non-IBD (IRR 1.82, 95\% CI 1.75-1.88). In adjusted Cox analysis, pneumonia risk remained increased for the IBD vs. non-IBD cohort (HR 1.54, 95\% CI 1.49-1.60), with increased risk in both CD (HR 1.71, 95\% CI 1.62-1.80) and UC (HR 1.41, 95\% CI 1.34-1.48). In the nested case-control analysis, use of biologic medications (OR 1.28, 95\% CI 1.08-1.52), corticosteroids (OR 3.62, 95\% CI 3.30-3.98) and proton pump inhibitor (PPI) (OR 1.14, 95\% CI 1.03-1.25) in the preceding 120 days were significantly associated with pneumonia.

Conclusions-Patients with IBD are at increased risk for pneumonia. Use of corticosteroids particularly increases pneumonia risk.

Corresponding Author: Millie D. Long MD, MPH, Campus Box 7080, University of North Carolina at Chapel Hill, Chapel Hill, NC 27599-7080, millie_long@med.unc.edu, Phone: 919-843-5795, Fax: 919-966-6842.

The statements, findings, conclusions, views, and opinions contained and expressed in this article are based in part on data obtained under license from the following IMS Health Incorporated information service(s): IMS LifeLink ${ }^{\mathrm{TM}}$ Health Plan Claims Database (Start Year-End Year3), IMS Health Incorporated. All Rights Reserved. The statements, findings, conclusions, views, and opinions contained and expressed herein are not necessarily those of IMS Health Incorporated or any of its affiliated or subsidiary entities. 


\section{Introduction}

Patients with inflammatory bowel disease (IBD), particularly those treated with immunosuppression, are at increased risk for infectious complications. In fact, infectious complications are among the most common causes of death in patients with IBD. ${ }^{1}$ In the United States (US), community acquired pneumonia (CAP) is the leading infectious cause of death. ${ }^{2}$ The most common etiologic agent of CAP in the US is pneumococcal pneumonia. In 2004 , there were an estimated 866,000 cases of pneumococcal pneumonia in the US with estimated costs of 2.5 billion dollars. ${ }^{3}$

Little is known about the risk of pneumonia in IBD patients. In a recent study of death certificates, respiratory illness was identified as a common causes of mortality in IBD patients ${ }^{1}$ and bacterial pneumonia has been one of the most frequently reported complications in IBD clinical studies. ${ }^{4}$ In the rheumatoid arthritis (RA) population, pneumonia has been shown to be a major cause of morbidity and mortality. ${ }^{5,6}$ In these patients, prednisone use has been associated with an increased risk of pneumonia hospitalization. ${ }^{5}$ The anti-tumor necrosis factor-alpha (anti-TNF) agents have been associated with an increased overall risk of infection in some, ${ }^{7,8}$ but not all ${ }^{9}$ studies. AntiTNF agents have not been found to increase the risk of hospitalization for pneumonia in RA. ${ }^{5}$

To further evaluate these associations in the IBD population, we aimed to determine the risk of pneumonia in patients with IBD as compared to a non-IBD cohort. We also aimed to determine whether specific immunosuppressive medications increase the risk of pneumonia in patients with IBD.

\section{Methods}

We analyzed the procedural and outpatient pharmaceutical insurance claims contained in the LifeLink ${ }^{\mathrm{TM}}$ Health Plan Claims Database (IMS Health Inc, Norwalk, CT) for the period January 1, 1997 through December 31, 2009. This longitudinal, patient-level database has been used in previous epidemiologic studies of IBD. ${ }^{10,11}$ The source database contains enrollment information on over 60 million persons from over 98 health plans across the U.S. The included health care plans capture a geographically diverse sample from across the United States. Only health plans that submit data for all members are included in the database, ensuring complete data capture and representative samples. Prior studies have reported the IMS Health database to be representative of the national commercially insured population on a variety of demographic measures, including geographic region, age, gender, and health plan type. ${ }^{12}$

\section{Study Design}

We performed a retrospective cohort study to determine the overall risk of pneumonia in IBD patients compared with a non-IBD cohort and a nested case-control study to determine the independent effects of medication use (immunosuppressive, anti-TNF therapy and corticosteroids) on pneumonia risk among patients with IBD. A similar design has 
previously been used by our group ${ }^{10}$ and also by Gupta et al ${ }^{13}$ to evaluate the incidence of disorders in patients with IBD and the effects of various medications.

\section{Cohort Study}

Patient selection-All patients aged $<64$ years with at least 12 months of continuous health plan enrollment were eligible for inclusion in this analysis. We chose 64 as the upper age limit to avoid the possibility of missing data resulting from Medicare dual eligibility (which begins at age 65). Individuals were also required to have ongoing pharmacy coverage with their health plan in order to fully capture medication exposures. We identified cases of Crohn's disease (CD) and ulcerative colitis (UC) using a previously reported administrative definition expanded to include updated medications recently approved for IBD indications. ${ }^{14}$ The precise definition included patients with at least 3 health care contacts, on different days, associated with an International Classification of Diseases, 9th Revision, Clinical Modification (ICD-9) diagnosis code for CD (555.xx) or UC (556.xx), or patients with at least 1 claim for $\mathrm{CD}$ or $\mathrm{UC}$ and at least 1 pharmacy claim for any of the following medications: mesalamine, olsalazine, balsalazide, sulfasalazine, 6mercaptopurine, azathioprine, methotrexate, infliximab, adalimumab, certolizumab pegol, natalizumab and enteral budesonide. For patients who had claims for both CD and UC, disease assignment was made according to the majority of the last 9 claims or the majority of total claims if there were fewer than 9. We matched each IBD patient to 4 non-IBD subjects by age, gender, calendar time and U.S. census region. Region of the country was defined by standard regional definitions from the United States Census Bureau. We ensured that each non-IBD subject was enrolled during the same calendar time as the IBD subject in order to account for the seasonality of pneumonia outcomes. All individuals with any ICD-9 code for human immunodeficiency virus (HIV) (042-044.9) were excluded related to differences in susceptibility to infections.

Cohort lead time and follow-up_Each cohort member was required to have a minimum of 6 months of health plan enrollment prior to cohort entry. This "screening period" was used to assess potential confounders of interest. Additionally, anyone with a pneumonia claim in the last month of this "screening period" was excluded, as this was considered to represent a prevalent pneumonia. Each member of cohort was followed until the first diagnosis of pneumonia, or, if none, until censoring at the earlier of one of two events: discontinuation of primary or pharmacy insurance coverage, or age $>64$.

Assessment of outcome (pneumonia)—Pneumonia was defined as 1) any 1 ICD-9 code for bacterial pneumonia (481, 482.xx-483.xx, 485, 486) combined with a prescription for a systemic antibiotic or 2) any hospital confinement code accompanied by one of the above ICD-9 codes for bacterial pneumonia. For the first definition, the antibiotic prescription had to be filled in the time period of 1 week prior to 1 week after the pneumonia ICD-9 code. Antibiotic prescriptions for any of the following classes (any day supply) were included in the definition: macrolide class (erythromycin, clarithromycin, azithromycin), penicillin class (amoxicillin, ampicillin, oxacillin, penicillin, dicloxacillin, nafcillin, oxacillin, amoxicillin/clavulanate), $3^{\text {rd }}$ or $4^{\text {th }}$ generation fluroquinolone class (levofloxacin, moxifloxacin, gatifloxacin, gemifloxacin), $3^{\text {rd }}$ or $4^{\text {th }}$ generation cephalosporin class 
(cefdinir, cefixime, cefotaxime, cefpodoxime, ceftibuten, ceftizoxime, ceftriaxone, cefoperazone, ceftazidime, cefepime) or tetracycline class (tetracycline, minocycline, doxycycline). Of note, ciprofloxacin (a second generation fluoroquinolone) was not included as part of the pneumonia outcome, as this antibiotic is not a "respiratory fluoroquinolone"15 and is often used for an indication of IBD independent of infection. A similar definition for outpatient pneumonia consisting of medication dispensing and ICD-9 codes has been used in prior studies of community acquired pneumonia using administrative data. ${ }^{16} \mathrm{~A}$ more sensitive secondary definition of pneumonia consisting only of any one of the above ICD-9 codes for bacterial pneumonia was then investigated.

Assessment of exposures-All exposure assessment occurred during the 6 month "screening period" prior to cohort entry. Health care utilization was estimated in a continuous fashion by the total of days with at least one health care contact over the time period. Utilization has been measured in this fashion in prior pharmacoepidemiology studies. ${ }^{17}$ Comorbidities were assessed using the Quan update ${ }^{18}$ of the validated Deyo comorbidity index for administrative data. ${ }^{19}$ Medication use, including immunosuppressive and biologic medications, was assessed in the exposure period in an any/none fashion. Additionally, as proton pump inhibitor (PPI) use can be associated with IBD and also with pneumonia, any PPI use was also extracted during the exposure period.

Evaluation of an Alternate Cohort-In order to cross-validate the outcome of pneumonia within our dataset, we also investigated the risk of pneumonia in a separate cohort of individuals with diabetes mellitus as compared to a non-diabetic cohort, using the same methodology as that used in the IBD cohort. Diabetes was chosen as a reference population due to the known increased risk of pneumonia ${ }^{20}$ and death from pneumonia in this population. ${ }^{21}$ Diabetes was defined using any 2 separate ICD- 9 codes for diabetes (250-250.7).

Statistical analysis: We used descriptive statistics to summarize characteristics of patients with and without IBD. Continuous variables are reported as mean $+/-$ standard deviation or median and interquartile range (IQR), and categorical variables are reported as percentages. We then calculated incidence rates of pneumonia (per 100,000 person-years) and used incidence rate ratios (IRRs) and 95\% confidence intervals (CIs) to compare the incidence of pneumonia in IBD and non-IBD patients. We also performed subsequent analyses, stratifying by region of the country (defined by United States census groups),age (in decades), and disease type (UC vs CD). To control for confounding, we assessed for possible violation of the proportional hazards assumption via log-log plots. As the assumption was not violated, we used Cox proportional hazards models to calculate hazard ratios (HRs) and 95\% CIs for the risk of pneumonia in the IBD cohort as compared to the non-IBD cohort, controlling for health care utilization and comorbidities. Analyses were repeated stratified by $\mathrm{CD}$ versus $\mathrm{UC}$ and within the alternate diabetes cohort. 


\section{Nested Case-Control Study}

We next conducted a case-control study evaluating the association between corticosteroids, immunosuppressive or biologic anti-TNF medication use and pneumonia. This study was nested within the previously defined cohort of patients with IBD.

Selection of cases and controls-Cases were those IBD patients who were diagnosed with pneumonia, and controls were those IBD patients without pneumonia. Each case patient was matched on gender, age, geographic region, disease type (CD or UC), and duration of follow-up to 4 IBD patients who did not have pneumonia using incidence density sampling. In this sampling technique, a case patient can also be a control patient, provided that the case patient has not yet been diagnosed with pneumonia (has not yet become a case) at the time he or she is selected as a control. Once a case is diagnosed with pneumonia, he or she is no longer eligible to become a control, or a case again. A total of 756 patients were included as both cases and controls. Each control was assigned an index date identical to the matched case's date of pneumonia, in order to account for seasonality.

Assessment of outcome (pneumonia)-The outcome was outpatient or inpatient pneumonia, as defined within the cohort study by ICD-9 codes, antibiotic prescriptions and hospital confinement codes.

\section{Assessment of exposures}

Medication Use: The primary medications evaluated included azathioprine and 6mercaptopurine (thiopurine class), methotrexate, tacrolimus and cyclosporine (calcineurin class), mycophenolate mofetil, infliximab, adalimumab, and certolizumab pegol (biologic anti-TNF class) and systemic corticosteroids. PPI use was also assessed, as this could be associated with both exposure and outcome. Medication exposures were analyzed with respect to the amount of time preceding the diagnosis of pneumonia in cases or the corresponding index date in controls. All exposures were defined as at least 1 outpatient pharmacy claim occurring during the 120 days prior to pneumonia diagnosis.

Potential confounders: Data on utilization and comorbidities were also included as exposure variables in the case-control study. These variables were assessed during the 6 month exposure period prior to pneumonia diagnosis. Utilization was defined in a continuous fashion as total number of days with at least one health care contact. Comorbidities were assessed through the Quan update ${ }^{18}$ of the validated Deyo comorbidity index ${ }^{19}$ for administrative claims data.

Statistical analysis: Characteristics of cases and controls were described using descriptive statistics. We then used conditional logistic regression to calculate odds ratios (ORs) and 95\% CIs for each medication exposure related to the outcome of pneumonia. We constructed a full model by using all potential confounders and eliminated these confounders via a backwards elimination strategy by using a change in estimate approach (threshold of $<10 \%$ change). Analyses were performed for patients with IBD and also stratified by $\mathrm{UC}$ and $\mathrm{CD}$ diagnosis. 


\section{Results}

The cohort study population included 108,604 patients with IBD. Of these, 50,932 had CD, 56403 had UC and 1,269 had IBD with unknown type. There were a total of 434,416 individuals in the non-IBD comparison cohort. The median length of follow up within this cohort was 24 months (IQR 12-42) with a range from 1-138 months after the 6 month "screening" period. Length of follow-up was similar for CD (34 months, IQR 19-51) and UC populations (36 months, IQR 21-54), while significantly less in the non-IBD comparison cohort (21 months, IQR 10-38). Characteristics of the patients with IBD as compared to the non-IBD cohort are shown in table 1 . There was increased health care utilization, immunosuppressive medication use and PPI use among the IBD cohort as compared to the matched non-IBD cohort, as expected. These same factors were increased in those with $\mathrm{CD}$ as compared to those with UC. Among the IBD cohort, there were also slightly higher rates of liver disease and COPD as compared to the non-IBD cohort.

For patients with IBD, the overall annual incidence of pneumonia was $138 / 10,000$, compared to 76/10,000 in the non-IBD cohort (IRR $1.8295 \%$ CI 1.75-1.88) (figure 1). The risk was somewhat greater for CD as compared to non-IBD (IRR 2.05 95\% CI 1.94-2.15) than for UC as compared to non-IBD (IRR $1.6295 \%$ CI 1.54-1.71). The incidence of pneumonia was then evaluated in strata of age, with the highest incidence in the 60+ age strata $(226 / 10,000)$. On adjusted Cox analysis, controlling for comorbidities, health care utilization, and PPI use, the relative risk remained elevated in the overall IBD population (HR 1.54, 95\% CI 1.49-1.60). Compared to non-IBD controls, the risk was greater for CD (HR 1.71, 95\% CI 1.62-1.80) than for UC (HR 1.41, 95\% CI 1.34-1.48). Analyses were also performed using the alternate definition of pneumonia (any 1 ICD-9 code). The effect size estimate remained similar with this alternate definition, with an IRR of $1.83,95 \%$ CI 1.78-1.89 for pneumonia risk in the IBD cohort as compared to the non-IBD cohort.

In the alternate diabetes cohort, the risk of pneumonia in diabetics as compared to nondiabetics was elevated to a similar extent (IRR 2.26, 95\% CI 2.17-2.35). The overall incidence of pneumonia among diabetics was $178 / 10,000$ as compared to $79 / 10,000$ in the non-diabetic cohort. In adjusted analyses, adjusting for health care utilization and comorbidities, the risk estimate remained similar (HR 1.84, 95\% CI 1.76-1.93).

In the nested case control study, 4856 IBD patients with pneumonia were matched to 18928 IBD patients without pneumonia. The characteristics of the populations are shown in table 2 . Patients with pneumonia had significantly more comorbidities, particularly COPD. Those with pneumonia also had significantly higher health care utilization and immunosuppressive medication use. PPI use was also associated with pneumonia. In the adjusted analyses, corticosteroid use (OR 3.62, 95\% CI 3.30-3.98), biologic use (OR 1.28, 95\% CI 1.08-1.52) and PPI use (OR 1.14, 95\% CI 1.03-1.25) were independently associated with pneumonia in the overall IBD population. Interestingly, calcineurin inhibitor use was associated with lower risk. Similar risk estimates were seen when stratified by CD or UC. Crude and adjusted analyses are shown in table 3 for specific medication use in the overall population, and by IBD subtype. 


\section{Discussion}

In this large cohort of commercially insured Americans, we have demonstrated an increased risk of pneumonia in patients with IBD as compared to the general population, with an adjusted effect estimate of HR 1.54, 95\% CI 1.49-1.60. Additionally, we found a particularly increased risk of pneumonia among patients on corticosteroids (OR OR 3.62, 95\% CI 3.30-3.98), a mild to moderate increased risk in patients on anti-TNF agents (OR 1.28, 95\% CI 1.08-1.52), and no increased risk associated with other classes of IBD medications.

Streptococcus pneumoniae is the most common cause of community acquired pneumonia (CAP), accounting for about $36 \%$ of cases of CAP in the general population. Vaccination against invasive pneumococcal disease is currently available in the US for both children and adults. Pneumococcal vaccine is recommended in IBD immunization guidelines. ${ }^{22}$ Therefore, studying pneumonia is important in IBD, as 1) pneumonia is associated with substantial costs, morbidity and mortality and 2) complications from pneumonia may be preventable through vaccination.

In this large cohort of commercially insured Americans, we have demonstrated an increased risk of pneumonia in patients with IBD as compared to the general population, with an adjusted effect estimate of HR 1.54, 95\% CI 1.49-1.60. Additionally, we found a particularly increased risk of pneumonia among patients on corticosteroids, a mild to moderate increased risk in patients on anti-TNF agents (OR 1.28, 95\% CI 1.08-1.52), and no increased risk associated with other classes of IBD medications. These findings are particularly noteworthy, considering that pneumonia is 1) associated with substantial costs, morbidity and mortality, and 2) often preventable, given that vaccination against Streptococcus pneumonia, the most common cause of community acquired pneumonia (CAP) accounting for about $36 \%$ of cases of CAP, is currently available for both children and adults.

To our knowledge, this is the first epidemiological study of the incidence and medicationspecific risk factors for pneumonia in patients with IBD. However, these findings are of similar direction and magnitude to prior studies in the RA population. Wolfe et al demonstrated an increased risk for pneumonia hospitalization (HR 1.7, 95\% CI 1.5-2.0) for patients with RA on corticosteroids, including a dose related increase in risk. ${ }^{5}$ Coyne et al found the overall annual incidence of lower respiratory tract infection (LRTI) in patients with RA was $2.3 \%$ with a mortality rate of $22.5 \%$. Corticosteroids were also associated with an increased risk of hospital admission with LRTI in this study. ${ }^{6}$ The anti-TNF agents have been associated with increased risk of hospitalization for infection in patients with RA, ${ }^{8}$ while other disease modifying agents (DMARDs) such as methotrexate were not. Other studies have not found an increased risk of serious hospitalized infection associated with the anti-TNF class, although there may be a relative increased risk with infliximab in the RA population. ${ }^{9}$

We also investigated the age-specific risks of pneumonia in patients with IBD, and found the highest relative risks in the younger ( $\leq 30$ years) group, with the highest absolute risk in the elderly (61-64). This is not dissimilar to the general population and the previously described studies of RA, ${ }^{5,6}$ where elderly individuals are at the highest risk for pneumonia. In the IBD 
population, the greatest absolute differences in mortality rates for IBD as compared to the general population also occur in the elderly. ${ }^{23}$ As in the general population, we also found that comorbidities, including COPD, were additional risk factors for pneumonia in the IBD population.

Our study has also confirmed two known associations, which help to substantiate the validity of our novel IBD-related findings. First, the relative risk of pneumonia in IBD, particularly for those with CD (HR 1.71, 95\% CI 1.62-1.80), was on par with the relative risk in a separate diabetes population (HR 1.84, 95\% CI 1.76-1.93). Other studies have found diabetes to be associated with an increased risk of pneumonia (HR 1.92, 95\% CI $1.84-1.99)^{20}$ and death from pneumonia (HR 1.67, 95\% CI 1.45-1.92). ${ }^{21}$ Additionally, recent data show an increased risk of pneumonia associated with PPI use. The mechanism behind this increased risk is hypothesized to be overgrowth of bacteria due to acid suppression in the stomach, which may increase the risk of micro-aspiration of bacteria, and therefore pneumonia. ${ }^{24} \mathrm{We}$ found an increased risk of pneumonia associated with PPI use in our IBD population (unadjusted OR 2.21, 95\% CI 2.04-2.39, adjusted OR 1.14, 95\% CI 1.03-1.25). Such findings are consistent with a recently published meta-analysis of pneumonia risk with PPI use (OR 1.36, 95\% CI 1.12-1.65). ${ }^{24}$

There are notable strengths to this study of pneumonia in IBD. We were able to include a large sample size. We also had great geographic diversity, with patients from all regions of the US. By drawing from a large number of health plans of varying size, type, and location, we believe these results to be broadly generalizable to the commercially insured US population. We obtained complete data on all billed outpatient prescriptions in order to completely capture medication exposures; rather than relying on patient recall. We were also able to account for health care utilization, as we had access to all health care contacts within the database. By studying an alternate cohort of patients with diabetes, who are known to have an increased risk of pneumonia, we were able to substantiate our outcome in a separate reference population. We were also able to account for other pharmacologic exposures, such as PPIs, that have been associated with pneumonia and may be more common among individuals with IBD. Finally, we were able to account for comorbidities through a validated comorbidity index for administrative data. ${ }^{18,19}$ This is particularly important, as comorbidities are known to be associated with pneumonia risk.

There are also several limitations to this study of administrative claims data. There is the possibility of misclassification of exposure and outcome related to the lack of clinical detail. We did use an IBD exposure definition previously used by our group ${ }^{10,11}$ that requires multiple health contacts and/or IBD related prescriptions. A similar, but even less specific, administrative case definition has been validated by Herrinton et al. The Herrinton definition was found to have a sensitivity exceeding $90 \%$ and a PPV exceeding $80 \%$ for overall IBD. ${ }^{25}$ We used an outcome definition of pneumonia that required both ICD-9 code and antibiotic dispensing. A similar definition of ICD-9 codes with antibiotic dispensing has been used in prior studies of pneumonia, ${ }^{16}$ although this definition has not been validated. We required both components (ICD-9 and medication dispensing), as prior validation studies of ICD-9 codes alone have been found to be imprecise. ${ }^{26}$ Any measurement bias in this definition would be non-differential by IBD or non-IBD status and would therefore bias our estimate 
towards the null value. We also performed a sensitivity analysis around the outcome definition by investigating a more sensitive definition consisting of any 1 ICD-9 code. The effect estimate for pneumonia risk with this definition did not change. Further support for the validity of the administrative definition used in this study is that the incidence of pneumonia in both the IBD and comparison cohorts was highest in the oldest age strata. Another limitation to this study is that the elderly (>age 65) and uninsured were not represented in our population. The lack of older persons in our study population does not affect the validity of the relative risks (IRRs, HRs, ORs) we presented in the context of the under 65 population. Finally, we were unable to account for certain exposures that may be related to the outcome, particularly smoking status. We were able to control for chronic obstructive pulmonary disease (COPD), which has been used as a proxy for smoking status in other studies using administrative data.

In conclusion, we believe that the increased risk of pneumonia in patients with IBD, particularly those treated with corticosteroids, should be viewed as a call to action. Pneumococcal vaccination is safe, effective, widely available, and known to prevent complications of pneumococcal pneumonia. Unfortunately, current vaccination efforts in the IBD population are lagging. In one tertiary care setting, only $9 \%$ of patients with IBD reported pneumococcal vaccination. ${ }^{27}$ As response to vaccination can be impaired with immunosuppression, ${ }^{28}$ the optimal time to vaccinate individuals with IBD is likely at diagnosis. Knowing risk factors for pneumonia in the IBD population, such as age and comorbidities, may increase awareness and promote prevention and/or early treatment. Additionally, awareness of the medication-specific risk factors: corticosteroids, anti-TNF agents and PPIs, may provide for timely intervention and ultimately improve outcomes.

\section{Acknowledgments}

Grant support: This work was supported by a Career Development Award from the Crohn's and Colitis Foundation of America (MDL), NIH P30 DK34987 (RSS), and NIH 1K08DK088957-01 (MDK).

\section{References}

1. Hutfless SM, Weng X, Liu L, Allison J, Herrinton LJ. Mortality by medication use among patients with inflammatory bowel disease, 1996-2003. Gastroenterology. 2007; 133:1779-86. [PubMed: 18054550]

2. Ruhnke GW, Coca-Perraillon M, Kitch BT, Cutler DM. Trends in mortality and medical spending in patients hospitalized for community-acquired pneumonia: 1993-2005. Med Care. 2010; 48:1111-6. [PubMed: 21063230]

3. Huang SS, Johnson KM, Ray GT, Wroe P, Lieu TA, Moore MR, Zell ER, Linder JA, Grijalva CG, Metlay JP, Finkelstein JA. Healthcare utilization and cost of pneumococcal disease in the United States. Vaccine. 2011; 29:3398-412. [PubMed: 21397721]

4. Colombel JF, Loftus EV Jr. Tremaine WJ, Egan LJ, Harmsen WS, Schleck CD, Zinsmeister AR, Sandborn WJ. The safety profile of infliximab in patients with Crohn's disease: the Mayo clinic experience in 500 patients. Gastroenterology. 2004; 126:19-31. [PubMed: 14699483]

5. Wolfe F, Caplan L, Michaud K. Treatment for rheumatoid arthritis and the risk of hospitalization for pneumonia: associations with prednisone, disease-modifying antirheumatic drugs, and anti-tumor necrosis factor therapy. Arthritis Rheum. 2006; 54:628-34. [PubMed: 16447241]

6. Coyne P, Hamilton J, Heycock C, Saravanan V, Coulson E, Kelly CA. Acute lower respiratory tract infections in patients with rheumatoid arthritis. J Rheumatol. 2007; 34:1832-6. [PubMed: 17659759] 
7. Furst DE. The Risk of Infections with Biologic Therapies for Rheumatoid Arthritis. Semin Arthritis Rheum. 2008

8. Lane MA, McDonald JR, Zeringue AL, Caplan L, Curtis JR, Ranganathan P, Eisen SA. TNF-alpha antagonist use and risk of hospitalization for infection in a national cohort of veterans with rheumatoid arthritis. Medicine (Baltimore). 2011; 90:139-45. [PubMed: 21358439]

9. Grijalva CG, Chen L, Delzell E, Baddley JW, Beukelman T, Winthrop KL, Griffin MR, Herrinton LJ, Liu L, Ouellet-Hellstrom R, Patkar NM, Solomon DH, Lewis JD, Xie F, Saag KG, Curtis JR. Initiation of tumor necrosis factor-alpha antagonists and the risk of hospitalization for infection in patients with autoimmune diseases. JAMA. 2011; 306:2331-9. [PubMed: 22056398]

10. Long MD, Herfarth HH, Pipkin C, Porter CQ, Sandler RS, Kappelman M. Increased Risk for NonMelanoma Skin Cancer in Patients with Inflammatory Bowel Disease. Clin Gastroenterol Hepatol. 2009

11. Long MD, Porter CQ, Sandler RS, Kappelman MD. Suboptimal Rates of Cervical Testing Among Women With Inflammatory Bowel Disease. Clin Gastroenterol Hepatol. 2008

12. Stempel DA, Mauskopf J, McLaughlin T, Yazdani C, Stanford RH. Comparison of asthma costs in patients starting fluticasone propionate compared to patients starting montelukast. Respir Med. 2001; 95:227-34. [PubMed: 11266241]

13. Gupta G, Lautenbach E, Lewis JD. Incidence and risk factors for herpes zoster among patients with inflammatory bowel disease. Clin Gastroenterol Hepatol. 2006; 4:1483-90. [PubMed: 17162240]

14. Kappelman MD, Rifas-Shiman SL, Kleinman K, Ollendorf D, Bousvaros A, Grand RJ, Finkelstein JA. The prevalence and geographic distribution of Crohn's disease and ulcerative colitis in the United States. Clin Gastroenterol Hepatol. 2007; 5:1424-9. [PubMed: 17904915]

15. Watkins RR, Lemonovich TL. Diagnosis and management of community-acquired pneumonia in adults. Am Fam Physician. 2011; 83:1299-306. [PubMed: 21661712]

16. Carrie AG, Kozyrskyj AL. Disease, temporal and sociodemographic influences on initial treatment of community-acquired pneumonia in Manitoba, Canada. Int J Antimicrob Agents. 2006; 28:95100. [PubMed: 16843645]

17. Lund JL, Sturmer T, Porter CQ, Sandler RS, Kappelman MD. Thiazolidinedione use and ulcerative colitis-related flares: an exploratory analysis of administrative data. Inflamm Bowel Dis. 2011; 17:787-94. [PubMed: 20848530]

18. Quan H, Sundararajan V, Halfon P, Fong A, Burnand B, Luthi JC, Saunders LD, Beck CA, Feasby TE, Ghali WA. Coding algorithms for defining comorbidities in ICD-9-CM and ICD-10 administrative data. Med Care. 2005; 43:1130-9. [PubMed: 16224307]

19. Deyo RA, Cherkin DC, Ciol MA. Adapting a clinical comorbidity index for use with ICD-9-CM administrative databases. J Clin Epidemiol. 1992; 45:613-9. [PubMed: 1607900]

20. Ehrlich SF, Quesenberry CP Jr. Van Den Eeden SK, Shan J, Ferrara A. Patients diagnosed with diabetes are at increased risk for asthma, chronic obstructive pulmonary disease, pulmonary fibrosis, and pneumonia but not lung cancer. Diabetes Care. 2010; 33:55-60. [PubMed: 19808918]

21. Seshasai SR, Kaptoge S, Thompson A, Di Angelantonio E, Gao P, Sarwar N, Whincup PH, Mukamal KJ, Gillum RF, Holme I, Njolstad I, Fletcher A, Nilsson P, Lewington S, Collins R, Gudnason V, Thompson SG, Sattar N, Selvin E, Hu FB, Danesh J. Diabetes mellitus, fasting glucose, and risk of cause-specific death. N Engl J Med. 2011; 364:829-41. [PubMed: 21366474]

22. Sands BE, Cuffari C, Katz J, Kugathasan S, Onken J, Vitek C, Orenstein W. Guidelines for immunizations in patients with inflammatory bowel disease. Inflamm Bowel Dis. 2004; 10:67792. [PubMed: 15472534]

23. Card T, Hubbard R, Logan RF. Mortality in inflammatory bowel disease: a population-based cohort study. Gastroenterology. 2003; 125:1583-90. [PubMed: 14724808]

24. Johnstone J, Nerenberg K, Loeb M. Meta-analysis: proton pump inhibitor use and the risk of community-acquired pneumonia. Aliment Pharmacol Ther. 2010; 31:1165-77. [PubMed: 20222914]

25. Herrinton LJ, Liu L, Lafata JE, Allison JE, Andrade SE, Korner EJ, Chan KA, Platt R, Hiatt D, O'Connor S. Estimation of the period prevalence of inflammatory bowel disease among nine health plans using computerized diagnoses and outpatient pharmacy dispensings. Inflamm Bowel Dis. 2007; 13:451-61. [PubMed: 17219403] 
26. Aronsky D, Haug PJ, Lagor C, Dean NC. Accuracy of administrative data for identifying patients with pneumonia. Am J Med Qual. 2005; 20:319-28. [PubMed: 16280395]

27. Melmed GY, Ippoliti AF, Papadakis KA, Tran TT, Birt JL, Lee SK, Frenck RW, Targan SR, Vasiliauskas EA. Patients with inflammatory bowel disease are at risk for vaccine-preventable illnesses. Am J Gastroenterol. 2006; 101:1834-40. [PubMed: 16817843]

28. Melmed GY, Agarwal N, Frenck RW, Ippoliti AF, Ibanez P, Papadakis KA, Simpson P, BaroletGarcia C, Ward J, Targan SR, Vasiliauskas EA. Immunosuppression impairs response to pneumococcal polysaccharide vaccination in patients with inflammatory bowel disease. Am J Gastroenterol. 105:148-54. [PubMed: 19755964] 


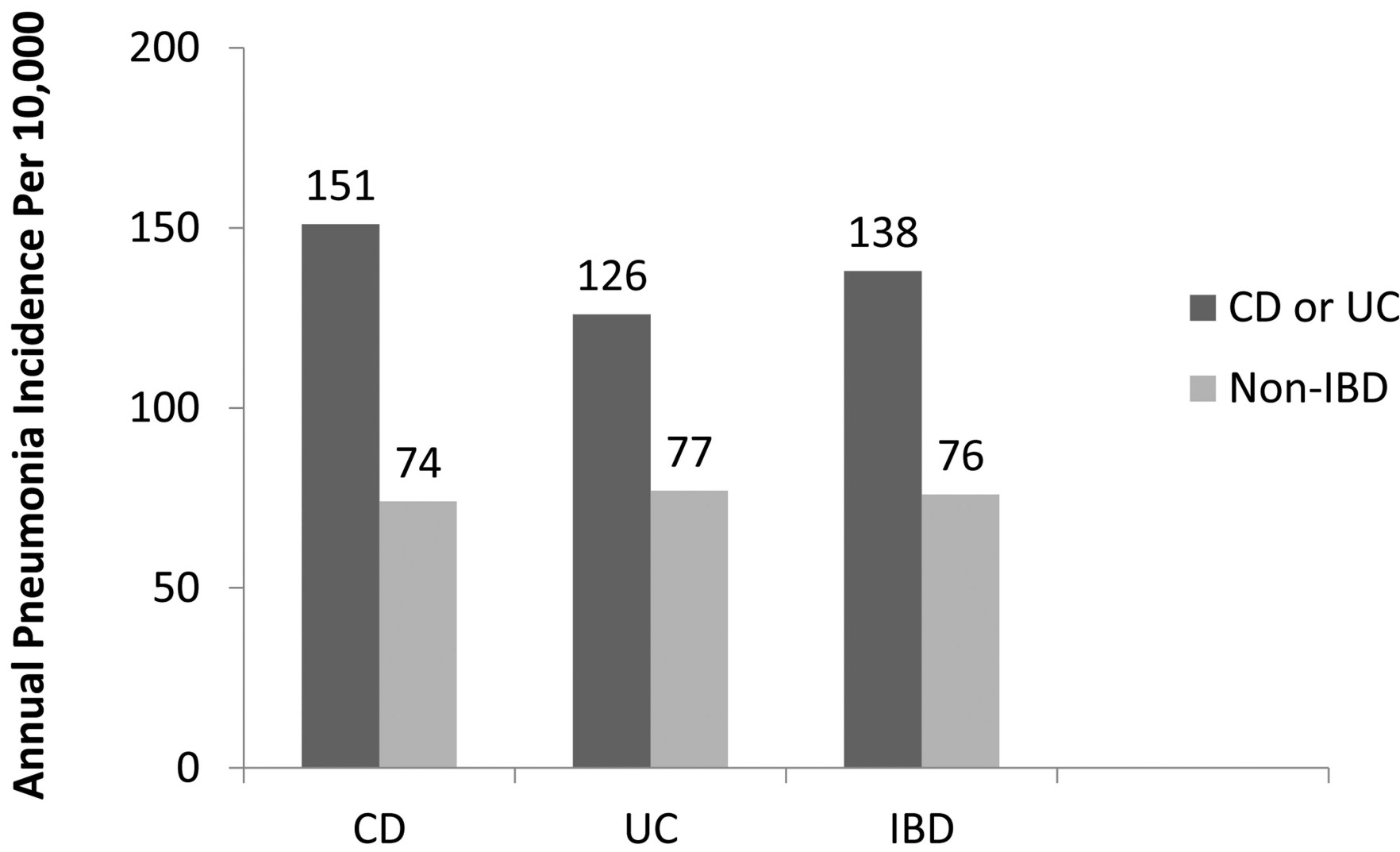

Figure 1.

Incidence of Recorded Pneumonia in IBD and non-IBD Populations 


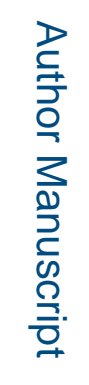

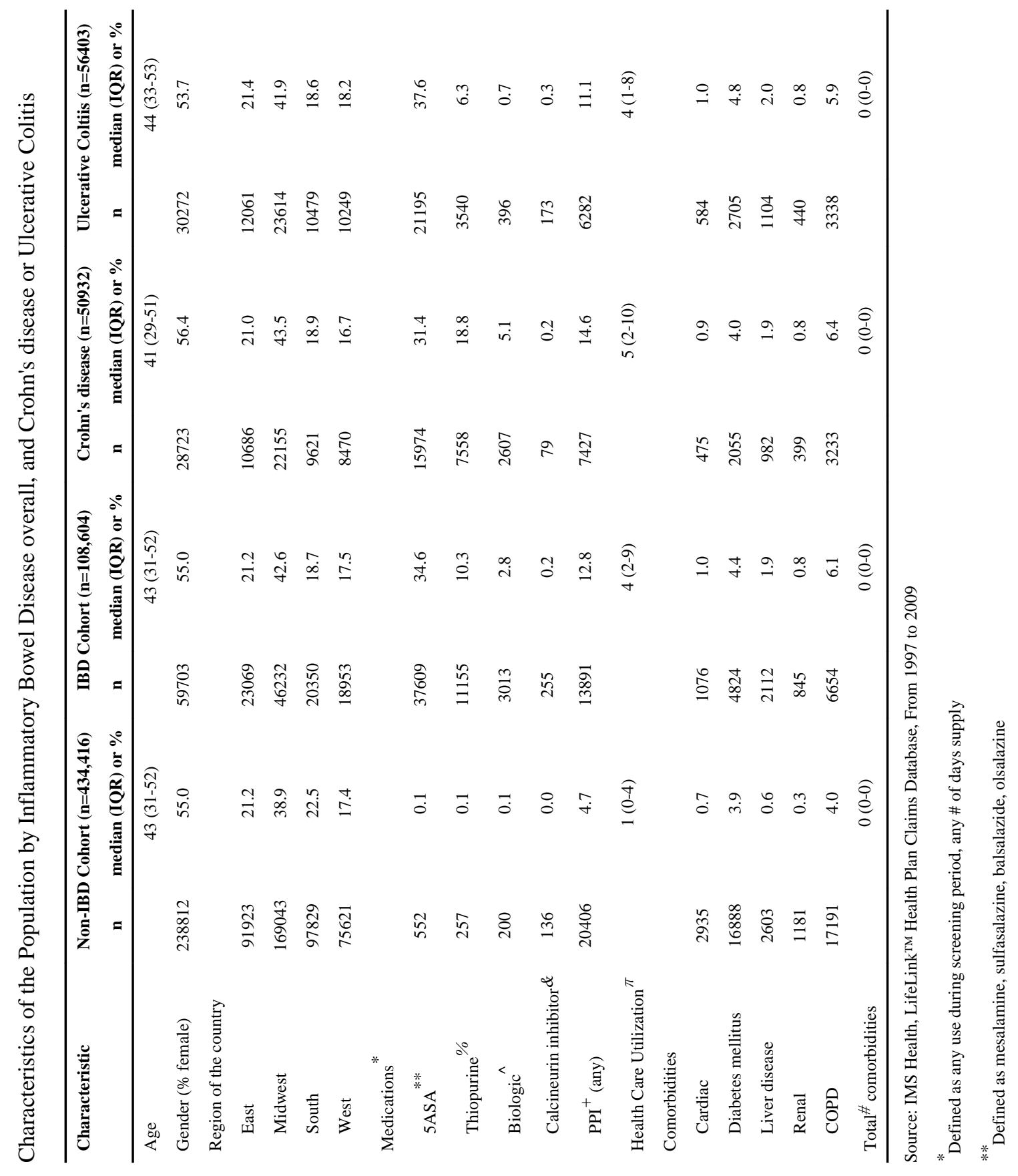



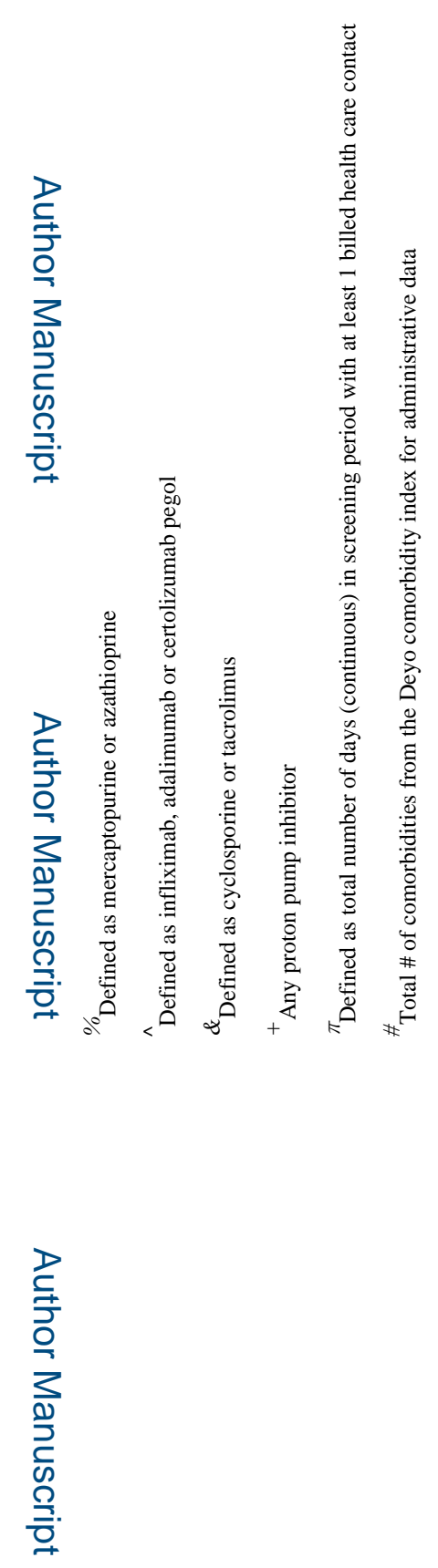

로을

Am J Gastroenterol. Author manuscript; available in PMC 2015 October 28. 


\section{Table 2}

Characteristics of the IBD Population by Pneumonia (matched characteristics include age, IBD type, gender, region)

\begin{tabular}{|c|c|c|c|c|c|}
\hline \multirow[t]{2}{*}{ Characteristics } & \multicolumn{2}{|c|}{ No Pneumonia (n=18928) } & \multicolumn{2}{|c|}{ Pneumonia $(n=4856)$} & \multirow[b]{2}{*}{ p value } \\
\hline & $\mathbf{n}$ & $\%$ or median (IQR) & $\mathbf{n}$ & \% or median (IQR) & \\
\hline $\operatorname{Age}^{* *}$ & 18928 & $47(35-55)$ & 4856 & $47(35-55)$ & 0.32 \\
\hline Gender ( $\%$ male $)$ & 8087 & 42.7 & 2083 & 42.9 & 0.83 \\
\hline \multicolumn{6}{|l|}{ IBD type } \\
\hline Crohn's disease & 9572 & 50.6 & 2453 & 50.5 & 0.04 \\
\hline Ulcerative colitis & 9276 & 49.0 & 2369 & 48.8 & \\
\hline Unknown & 80 & 0.4 & 341 & 0.7 & \\
\hline Utilization $\wedge$ & 18928 & $5(1-11)$ & 4856 & $13(7-24)$ & $<0.01$ \\
\hline \multicolumn{6}{|l|}{ Region } \\
\hline East & 3530 & 18.7 & 904 & 18.6 & 0.99 \\
\hline Midwest & 8649 & 45.7 & 2229 & 45.9 & \\
\hline South & 2858 & 15.1 & 731 & 15.1 & \\
\hline West & 3891 & 20.6 & 992 & 20.4 & \\
\hline \multicolumn{6}{|l|}{ Comorbidities } \\
\hline Cardiac & 317 & 1.7 & 352 & 7.3 & $<0.01$ \\
\hline Diabetes mellitus & 1219 & 6.4 & 596 & 12.3 & $<0.01$ \\
\hline Liver disease & 498 & 2.6 & 329 & 6.8 & $<0.01$ \\
\hline Renal & 226 & 1.2 & 205 & 4.2 & $<0.01$ \\
\hline COPD & 1423 & 7.5 & 1935 & 31.6 & $<0.01$ \\
\hline Rheumatologic & 401 & 2.1 & 288 & 5.9 & $<0.01$ \\
\hline \# Comorbidities $=$ & 18928 & $0(0-0)$ & 4856 & $1(0-1)$ & $<0.01$ \\
\hline \multicolumn{6}{|l|}{ Medications $\%$} \\
\hline Biologic $^{+}$ & 587 & 3.2 & 292 & 6.0 & $<0.01$ \\
\hline Thiopurine $\&$ & 1617 & 8.5 & 593 & 12.2 & $<0.01$ \\
\hline Calcineurin $^{\$}$ & 52 & 0.3 & 43 & 0.9 & $<0.01$ \\
\hline Corticosteroid & 1485 & 7.9 & 1531 & 31.5 & $<0.01$ \\
\hline 5-ASA & 4417 & 23.3 & 1314 & 27.1 & $<0.01$ \\
\hline PPI & 2471 & 13.1 & 1182 & 24.3 & $<0.01$ \\
\hline
\end{tabular}

Source: IMS Health, LifeLink ${ }^{\mathrm{TM}}$ Health Plan Claims Database, From 1997 to 2009

* p value by Pearson's chi square for categorical variables, Wilcoxon rank sum for continuous variables

** Age at time of pneumonia (case) or index date (control)

Defined as total number of days (continuous) with any health care contact over observation period

$=$ Number of comorbities from Charlson comorbidity index

${ }^{\%}$ Any use over observation period 
${ }^{+}$Infliximab, adalimumab or certolizumab pegol

$\&$ Mercaptopurine or azathioprine

$\$$ Cyclosporine or tacrolimus 


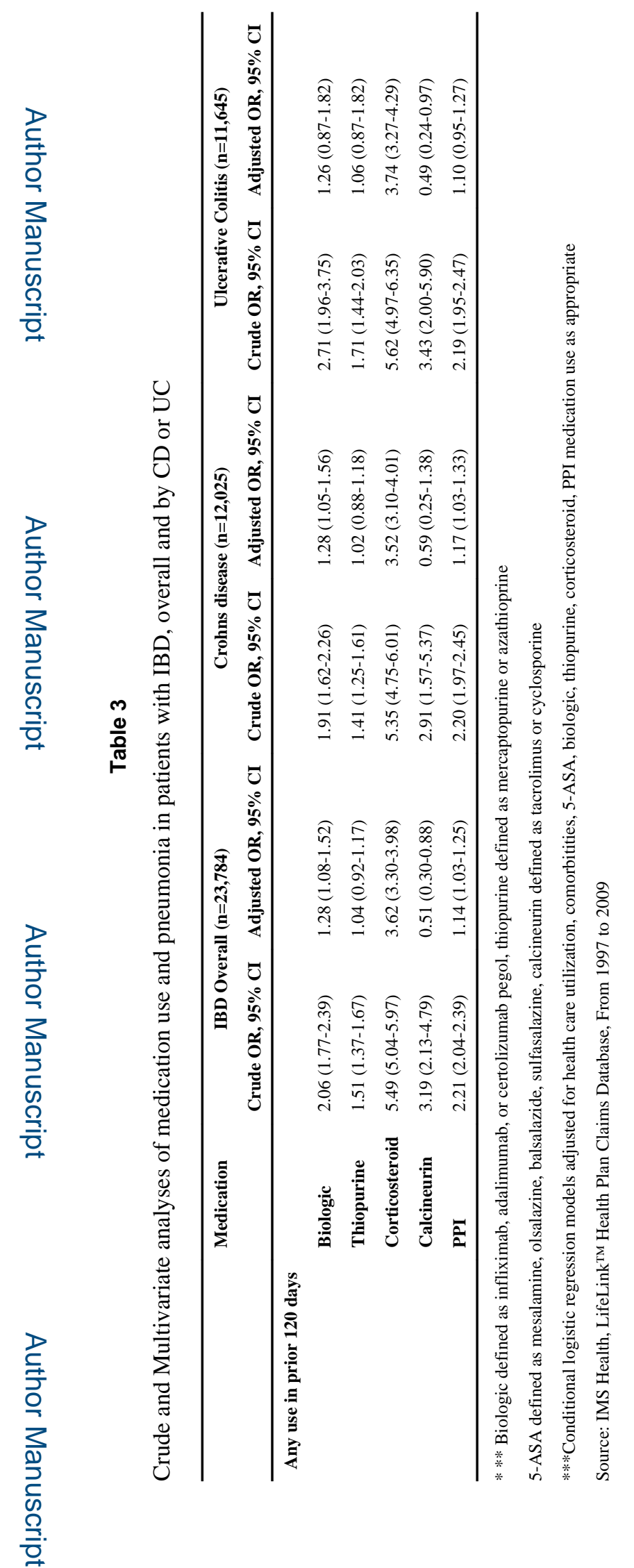

Am J Gastroenterol. Author manuscript; available in PMC 2015 October 28. 